\title{
Cross-Comparisons of Gait Speeds by Automatic Sensors and a Stopwatch to Provide Converting Formula Between Measuring Modalities
}

\author{
Hee-Won Jung ${ }^{1}$, Hyun-Chul Roh ${ }^{2}$, Sun-wook Kim ${ }^{3}$, Sunyoung $\mathrm{Kim}^{4}$, Miji Kim${ }^{5}$, Chang Won Won ${ }^{4}$ \\ ${ }^{1}$ Graduate School of Medical Science and Engineering, Korea Advanced Institute of Science and Technology, Daejeon, Korea \\ ${ }^{2}$ Dyphi Research Institute, Dyphi Inc., Daejeon, Korea \\ ${ }^{3}$ Internal Medicine, Ollin Clinic, Seongnam, Korea \\ ${ }^{4}$ Department of Family Medicine, Kyung Hee University Medical Center, College of Medicine, Kyung Hee University, Seoul, Korea \\ ${ }^{5}$ Department of Biomedical Science and Technology, College of Medicine, East-West Medical Research Institute, Kyoung Hee University, Seoul, \\ Korea
}

Corresponding Author:

Chang Won Won, MD, PhD

https://orcid.org/0000-0002-6429-4461

Department of Family Medicine,

Kyung Hee University Medical Center,

23 Kyungheedae-ro, Dongdaemun-gu,

Seoul 02447, Korea

E-mail: chunwon62@naver.com

Received: April 3, 2019

Revised: April 29, 2019

Accepted: April 30, 2019
Background: We aimed to compare 4 automatic devices with a conventional stopwatch for measuring gait speed. Methods: We used 4 experimental devices to automatically measure gait speed: 1) Gaitspeedometer (GSM) 1, with laser sensors; 2) GSM2, with ultrasound sensors; 3) GSM3, with infrared sensors; and 4) GSM4, with a light detection and ranging sensor. To assess compatibility between different versions of GSMs, we collected 426 data points from 4 young engineers walking at random speeds and with varying postures. We used these data to convert gait speed measured by GSM1 and 2 for compatibility with GSM3 in the Korean Frailty and Aging Cohort Study (KFACS) dataset. Results: Mean gait speeds measured with GSMs $1-4$ were $1.7 \%$ slower $\left(R^{2}=0.997\right), 12.2 \%$ faster $\left(R^{2}=0.993\right), 1.3 \%$ slower $\left(R^{2}=0.999\right)$, and $4.3 \%$ slower $\left(R^{2}=0.996\right)$, respectively, than the gait speed measured with a stopwatch. The concordance correlation coefficient between each GSM and the stopwatch was higher than 0.9. Using linear regression analysis with no constant term, conversion formulas for GSMs were established for the KFACS dataset using GSM1 and GSM2. Conclusion: The 4 methods of automatic gait speed measurement and the manually measured gait speed correlated well with each other, and we hope these new technologies reduce barriers to measuring older people's gait speed in busy clinical settings. (Ann Geriatr Med Res 2019;23:71-76)

Key Words: Walking speed, Screening, Diagnosis, Data analysis

\section{INTRODUCTION}

Growing evidence has shown the importance of physical performance in older adults as a parameter of future wellbeing without geriatric adverse outcomes. ${ }^{1)}$ Usual gait speed, a simple parameter that can be easily measured, has been considered a key screening measure for decreased physical performance in older people, and even called a functional vital sign. ${ }^{2-5)}$ Indeed, studies on older people in both clinical and research settings have shown the feasibility of measuring gait speed with varying protocols. From epidemiological studies, gait speed not only is considered to be indicative of the general health status of an individual but also can predict important clinical outcomes such as mobility disability, falls, cognitive impairment, institutionalization, and even mortality. ${ }^{1,6-10)}$ Furthermore, gait speed in clinical patients is predictive of geriatric adverse outcomes in patients undergoing surgical or medical procedures. ${ }^{11,12)}$
In usual clinical practice and research, gait speed has been measured with a stopwatch in a predefined distance, with or without acceleration and deceleration. In Asian populations, a $4-\mathrm{m}$ usual gait speed of less than $0.8 \mathrm{~m} /$ $\mathrm{s}$ measured with a stopwatch has been considered slow in both men and women, although there are some population-specific differences in the distribution of usual gait speed. ${ }^{13,14)}$ However, manually measured gait speed can be affected by inter- and intra-observer variations because the method depends on the interpretation of an observer determining when the examinee's foot or trunk has passed a predetermined point. ${ }^{15}$

For large-scale multicenter epidemiological and clinical studies, this inter-observer variation in gait speed measurements may affect the results of studies of gait speed parameters. Therefore, in an effort to reduce possible errors in measuring gait speed associated with observer factors and to reduce training requirements for research staff using stopwatch-based manual measurement of gait 
speed, we aimed to develop sensor-based instruments that can be easily used to produce reliable gait speed data. As a proof of concept, we studied the feasibility of automatic gait speed measurement in the outpatient clinic setting and found these parameters might be useful for screening frailty status. $^{16)}$

In the Korean Frailty and Aging Cohort Study (KFACS), a nationwide multicenter prospective cohort study of the clinical importance of frailty in Korea, we intended to assess the feasibility and validity of 3 different versions of gait speed-measuring instruments with distinct types of sensors (Gaitspeedometers, GSMs). Our goal was to develop a portable device that is readily available to use in field studies with reliable performance in measuring gait speeds of older people with varying degrees of physical performance. Because we changed sensor modalities once a year in response to real-world feedback regarding the device users' experiences during the previous year, we believed that cross-sensor comparisons were needed to deal with the accumulated gait speed data in this cohort study.
Therefore, in this study, we first aimed to compare 3 different types of gait speed measuring devices with the latest device using a light detection and ranging (LiDAR) sensor and the traditionally used stopwatch. Also, we aimed to provide converting equations for data acquired from these devices for future research using automatically measured gait speed data in the KFACS.

\section{MATERIALS AND METHODS}

\section{Development of GSM}

Dyphi Inc. (Daejeon, Korea) serially provided 4 types of GSMs, using different sensors. We first used a laser beam breaker system consisting of 2 laser beams and receivers (GSM1) installed $4 \mathrm{~m}$ apart (Figs. 1A and 2A). A clock in the microcontroller measured elapsed time, starting when the leading ankle crossed the first beam and finishing when the leading ankle crossed the second beam. This first instrument was difficult to use in field studies as the laser beam was narrow and straight with little margin of error
A

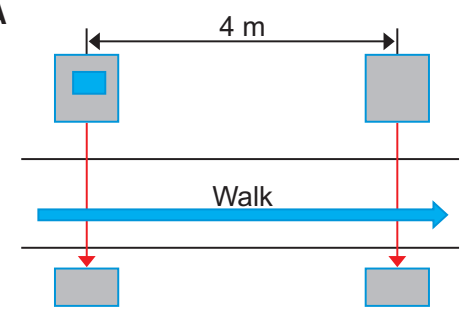

C

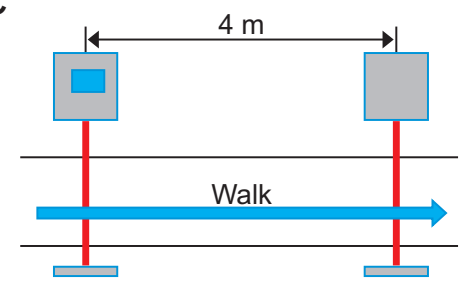

B

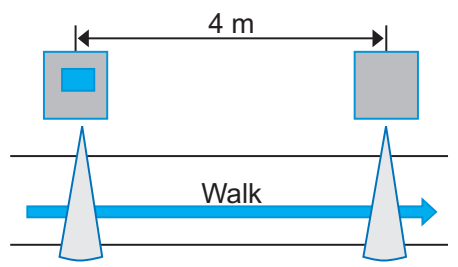

D

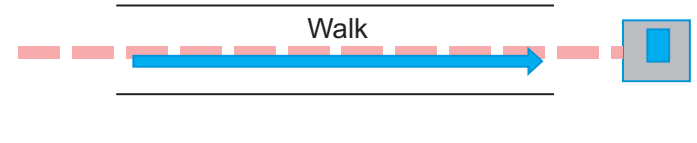

Fig. 1. Gait speed measuring layouts for Gaitspeedometers (GSMs). (A) GSM1, with 2 laser beams; (B) GSM2, with 2 ultrasound sensors; (C) GSM3, with 2 infrared sensors; (D) GSM4, with a light detection and ranging sensor.

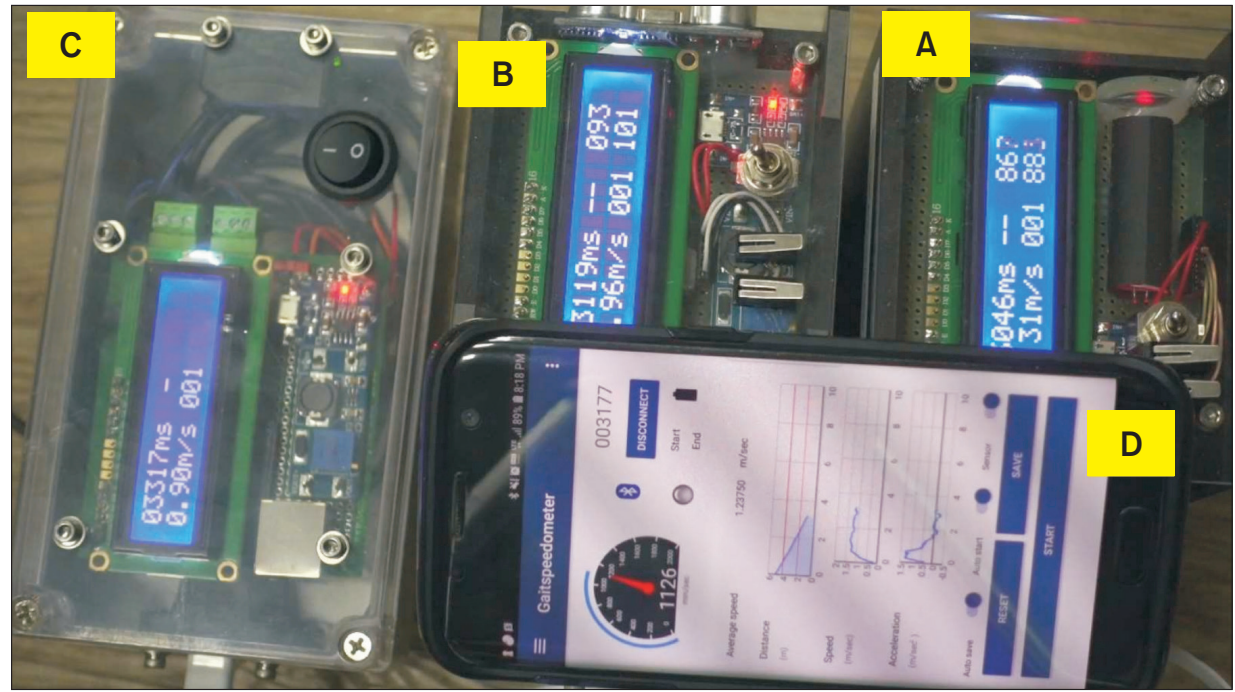

Fig. 2. In the cross-comparison study, 4 Gaitspeedometers (GSMs) were simultaneously used to measure gait speeds of participants. (A) GSM1; (B) GSM2; (C) GSM3; (D) GSM4. 
during installation. Therefore, Dyphi designed the next version, using 2 ultrasound distance sensors (GSM2; Figs. $1 \mathrm{~B}$ and $2 \mathrm{~B})$. Time measurement started and finished when the leading ankle entered the measuring angle $\left(15^{\circ}\right.$, horizontally) of the first and second ultrasound sensor, respectively. However, after realizing that the side of the leading leg at the start and finish may affect the result, causing up to a $10 \%$ shorter effective ranging distance due to the fan-beam effect, Dyphi developed the next version using industrial infrared sensors (GSM3; Figs. 1C and 2C). This modality used a beam width of $5 \mathrm{~cm}$ with a total reflection mirror, thereby ameliorating the difficulties of installation with the GSM1 but preserving accuracy comparable to a laser beam. Finally, Dyphi developed the GSM4 (Figs. 1D and 2D), which uses a single highly accurate one-dimensional LiDAR sensor measuring the longitudinal distance between the sensor and the trunk of the person walking with high spatial $( \pm 5 \mathrm{~cm})$ and time resolution $(10 \mathrm{~ms})$.

\section{Cross-comparison of 4 GSMs}

We simultaneously installed 4 versions of the GSM in a hallway and measured varying gait speeds of 4 young healthy engineers with these GSMs and a stopwatch. For manually measuring gait speed with stopwatch, we started and finished the measurement when the leading edge of the foot crossed the starting and finishing line, which was marked on the floor. A video camera recorded the displays of the GSMs (https://youtu.be/ydsRDkw34ro). We analyzed these data to compare the characteristics of the 4 GSMs.

In this cross-comparison study of 4 GSMs, 4 young healthy engineers (aged 29-34 years) were instructed to walk through a corridor in a random manner, including slow and fast paces. Gait speed was measured for $4 \mathrm{~m}$, with $1 \mathrm{~m}$ before and after the measured distance allowed for acceleration and deceleration.

\section{Producing Calibrated Gait Speed Data for KFACS}

We used gait speed data from the KFACS, a prospective cohort study started in 2016 targeting people aged 70-84 years to identify factors that may contribute to frailty in community-dwelling older adults. ${ }^{17,18)}$ In this study, we used gait speeds of 1,491 participants in 2016 whose gait speed was measured using GSM1 and 1,337 participants in 2017 whose gait speed was measured using both GSM2 and a stopwatch.

The KFACS protocol was approved by the Institutional Review Board (IRB) of the Kyung Hee University (IRB number: 2015-12-103), and written consent was obtained from participants before the initiation of the study. This study was waived for informed consents from review by the IRB of the Clinical Research Ethics Committee of the Kyung Hee University Medical Center (IRB number: 201903-041).

In KFACS, participants were asked to walk at their usual gait speed, and the elapsed time for a 4-m distance was measured twice and averaged, with unmeasured $1.5-\mathrm{m}$ ac- celeration and deceleration zones. In measuring gait speed for KFACS, GSM1 was used in 2016, GSM2 was used in 2017, and GSM3 has been used since 2018. As we aimed to standardize gait speed measurements in future research using the KFACS dataset, we established a formula for converting gait speeds measured by each GSM version in this cross-comparison study, to calibrate gait speeds to compatible with GSM3.

\section{Statistical Analysis}

We used linear regression analysis, Bland-Altman plots, and concordance correlation coefficients ${ }^{19)}$ to compare gait speed variables from GSMs and stopwatch. In comparing methods of gait speed measurements, we used linear regression analysis without a constant term, with an a priori hypothesis that methods would be free from offsets when compared to other methods, since measuring distance was fixed to $4 \mathrm{~m}$. To check for proportional bias, we used linear regression analysis using data points of Bland-Altman plots. ${ }^{20)}$ For statistical analysis, STATA 15 (StataCorp, College Station, TX, USA) was used and 2-sided p-values of $<0.05$ were considered significant.

\section{RESULTS}

\section{Cross-comparison of 4 GSMs}

We recorded 426 data points of gait speed measured using 4 types of GSM and a stopwatch. Generally, gait speed measured by the 4 types of GSMs correlated with the manually measured gait speed (Fig. 3A-D). According to linear regression analysis with no constant term, the mean gait speeds using GSM1-4 were 1.7\% slower $\left(\mathrm{R}^{2}=0.997\right), 12.2 \%$ faster $\left(R^{2}=0.993\right), 1.3 \%$ slower $\left(R^{2}=0.999\right)$, and $4.3 \%$ slower $\left(\mathrm{R}^{2}=0.996\right)$, respectively, than gait speeds measured using a stopwatch. Linear regression analyses using data points of Bland-Altman plots (Fig. 3E-H) revealed significant proportional biases for GSM2 (standardized $\beta=0.30, p<0.001$, $\left.\mathrm{R}^{2}=0.093\right)$ and GSM4 $\left(\beta=-0.63, \mathrm{p}<0.001, \mathrm{R}^{2}=0.392\right)$ compared with the stopwatch method. There was no significant proportional bias for GSM1 ( $\beta=0.07, p=0.155, R^{2}=0.005$ ) or GSM3 ( $\beta=-0.07, p=0.132, R^{2}=0.003$ ) compared with the stopwatch method. Concordance correlation coefficients $(\rho)$ for manually measured gait speeds were $0.979(p<0.001)$, $0.908(\mathrm{p}<0.001), 0.998(\mathrm{p}<0.001)$, and $0.982(\mathrm{p}<0.001)$ for GSM1-4, respectively.

From these data points, we established a conversion table for gait speed measured with GSM1-4 and with a stopwatch (Table 1), using linear regression analysis with no constant term. Every correlation coefficient $\left(R^{2}\right)$ between gait speed measured with GSM1-4 and a stopwatch was higher than 0.990, with p-values of $<0.001$.

\section{Calibrated Gait Speed Data for KFACS}

In 2016, usual gait speed was measured in 1,491 participants (mean age 76.1 years, standard deviation [SD] 3.9, $46.8 \%$ male) using GSM1. In 2017, usual gait speed was 
A

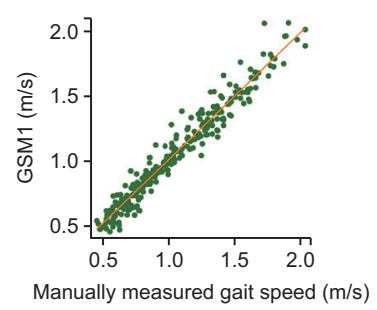

E

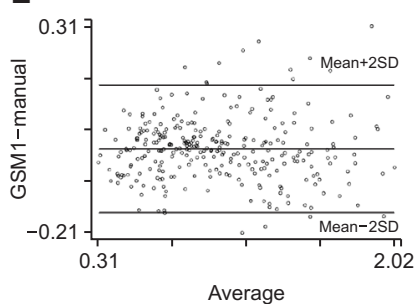

B

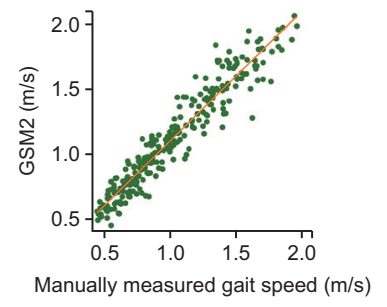

$\mathrm{F}$

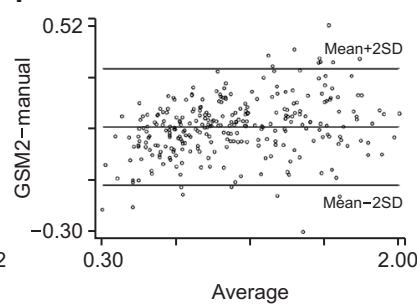

C

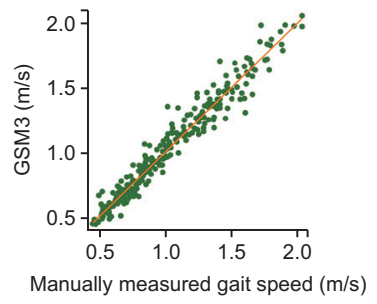

G

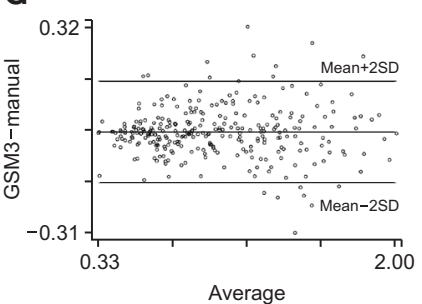

D

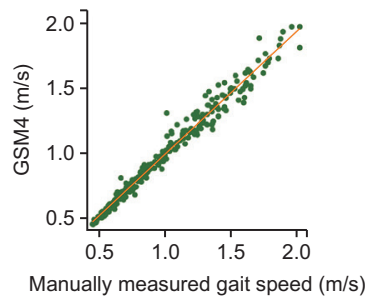

$\mathrm{H}$

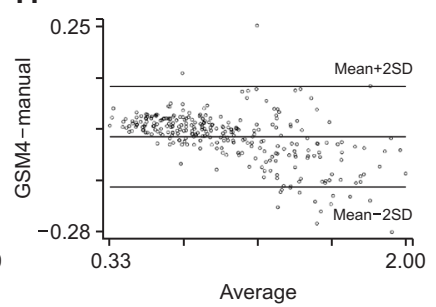

Fig. 3. (A-D) Scatterplots showing correlations between gait speeds measured using 4 types of Gaitspeedometers (GSMs) and a stopwatch. (E-H) Bland-Altman plots for gait speeds measured using 4 types of GSMs and a stopwatch. SD, standard deviation.

measured in 1,337 participants (mean age 75.7, SD 3.8, 47.5\% male) using GSM2.

Using the converting coefficients in Table 1, we transformed the gait speed data of 2016 and 2017 to be compatible with the gait speeds measured using GSM3. Before transformation, mean gait speeds measured in 2016 and 2017 were $1.12 \mathrm{~m} / \mathrm{s}$ (SD 0.27) and $1.18 \mathrm{~m} / \mathrm{s}$ (SD 0.26), respectively. After transformation to match GSM3, the respective adjusted mean gait speeds were $1.10 \mathrm{~m} / \mathrm{s}(\mathrm{SD} 0.27)$ and $1.10 \mathrm{~m} / \mathrm{s}$ (SD 0.24).

\section{DISCUSSION}

In this study, we found that 4 different modalities of automatic gait speed measurement and the traditional stopwatch-based method for gait speed measurement correlated with each other. High concordance rates were observed when each automatic method was compared with the stopwatch-based method, showing that gait speed data measured with an automatic sensor might be interpreted similarly, in terms of cutoff values for screening frailty and sarcopenia, to previous data established with stopwatches.

Even though GSM1-3 used the same scheme of beam breaking to measure gait speed, we found from this study that differences in the sensor mechanisms can affect measuring characteristics. For example, when gait speed was measured with GSM2 (Fig. 1B), a $15^{\circ}$ fanning effect of the ultrasound could reduce the effective distance of gait speed measurement when the left foot crosses the first beam and the right foot crosses the last beam. Indeed, from our study, we found that gait speed measured with GSM2 was faster than speeds measured with GSM1, 3, and 4 . This difference due to the fanning effect may be even higher if a participant has a wide-based gait ${ }^{211}$ or has a tendency to veer to the right during the gait speed examination. Therefore, in the future, sensor characteristics should be considered when designing measuring systems for human physical performance.

In contrast to GSM1-3 using a beam-breaking scheme, GSM4 used a high-frequency longitudinal distance measuring method with LiDAR technology. With the GSM4 sensor facing the participant's trunk, the distance between the GSM4 and the participant was measured and recorded every $10 \mathrm{~ms}$, and using these values, the one-dimensional time-dependent trajectory of the participant could be obtained. Measuring trunk speed with accelerometers has been examined previously, ${ }^{22)}$ but to our knowledge, our study was the first to study gait speed using LiDAR to assess the movement of the trunk. Although this scheme differed from the traditional approach of watching the participant's ankle crossing the designated marking on the floor or wall, results from GSM4 were highly correlated with gait speeds that were either manually measured or measured with GSM1-3. Given the similar characteristics in terms of gait speed measurements, advantages of the longitudinal one-dimensional LiDAR include the ability to obtain additional variables such as real-time acceleration and variations in gait velocities for future research that cannot be assessed with a stopwatch or beam breakers in older adults.

From the conversion table we produced by simultaneously using GSM1-4 and a stopwatch, we could calibrate gait speed data measured with GSM1 and -2 in 2016 and 2017, respectively. Interestingly, when gait speeds measured using GSM1 and -2 were converted to match the characteristics of GSM3-measured gait speeds, the overall mean gait speeds of 2016 and 2017 became identical. Although GSM3-based gait speed data are not currently available in the KFACS, calibrated gait speed data from 
Table 1. Converting coefficients (multipliers) calculated for gait speeds measured with GSM1-4 and a stopwatch using linear regression analysis with no constant term

\begin{tabular}{|c|c|c|c|c|c|}
\hline Devices & From stopwatch & From GSM1 & From GSM2 & From GSM3 & From GSM4 \\
\hline To stopwatch & 1.000 & 1.017 & 0.878 & 1.014 & 1.043 \\
\hline To GSM1 & 0.980 & 1.000 & 0.930 & 1.007 & 1.053 \\
\hline To GSM2 & 1.131 & 1.072 & 1.000 & 1.070 & 1.151 \\
\hline To GSM3 & 0.986 & 0.988 & 0.929 & 1.000 & 1.046 \\
\hline To GSM4 & 0.955 & 0.947 & 0.863 & 0.952 & 1.000 \\
\hline
\end{tabular}

GSM, Gaitspeedometer.

GSM1 and -2 provide evidence-based support for merging GSM3-based data in the future.

Although this study was the first to compare gait speed measurements with GSM1-4 and a stopwatch, there are several limitations. First, this was a cross-comparison study of methods in young healthy engineers rather than in community-dwelling older adults with varying states of physical performance. However, by simultaneously using 5 methods and having the participants walk at random speeds, we acquired a wide array of gait speeds ranging from 0.5 to $2.0 \mathrm{~m} / \mathrm{s}$. Second, because a single researcher (HWJ) measured all 426 walks using a stopwatch, interobserver effects of manually measured gait speed could not be assessed.

In conclusion, all 4 types of GSMs showed concordance with each other and the stopwatch for measuring gait speed. These results may provide evidence-based guidance for researchers interpreting gait speed data from different types of GSMs in future research.

\section{CONFLICTS OF INTEREST DISCLOSURES}

The researchers claim no conflicts of interest.

\section{REFERENCES}

1. Perera S, Patel KV, Rosano C, Rubin SM, Satterfield S, Harris T, et al. Gait speed predicts incident disability: a pooled analysis. J Gerontol A Biol Sci Med Sci 2016;71:63-71.

2. Middleton A, Fritz SL, Lusardi M. Walking speed: the functional vital sign. J Aging Phys Act 2015;23:314-22.

3. Fritz S, Lusardi M. White paper: "walking speed: the sixth vital sign”. J Geriatr Phys Ther 2009;32:46-9.

4. Hardy SE, Perera S, Roumani YF, Chandler JM, Studenski SA. Improvement in usual gait speed predicts better survival in older adults. J Am Geriatr Soc 2007;55:1727-34.

5. Studenski S, Perera S, Wallace D, Chandler JM, Duncan PW, Rooney E, et al. Physical performance measures in the clinical setting. J Am Geriatr Soc 2003;51:314-22.

6. Rosso AL, Verghese J, Metti AL, Boudreau RM, Aizenstein HJ, Kritchevsky S, et al. Slowing gait and risk for cognitive impairment: the hippocampus as a shared neural substrate. Neurology 2017;89:336-42.

7. Best JR, Liu-Ambrose T, Boudreau RM, Ayonayon HN, Satterfield S, Simonsick EM, et al. An evaluation of the longitudinal, bidi- rectional associations between gait speed and cognition in older women and men. J Gerontol A Biol Sci Med Sci 2016;71:1616-23.

8. Studenski S, Perera S, Patel K, Rosano C, Faulkner K, Inzitari M, et al. Gait speed and survival in older adults. JAMA 2011;305:50-8.

9. Brach JS, Studenski SA, Perera S, VanSwearingen JM, Newman $\mathrm{AB}$. Gait variability and the risk of incident mobility disability in community-dwelling older adults. J Gerontol A Biol Sci Med Sci 2007;62:983-8.

10. Jung HW, Jang IY, Lee CK, Yu SS, Hwang JK, Jeon C, et al. Usual gait speed is associated with frailty status, institutionalization, and mortality in community-dwelling rural older adults: a longitudinal analysis of the aging study of Pyeongchang rural area. Clin Interv Aging 2018;13:1079-89.

11. Afilalo J, Mottillo S, Eisenberg MJ, Alexander KP, Noiseux N, Perrault LP, et al. Addition of frailty and disability to cardiac surgery risk scores identifies elderly patients at high risk of mortality or major morbidity. Circ Cardiovasc Qual Outcomes 2012;5:222-8.

12. Afilalo J, Eisenberg MJ, Morin JF, Bergman H, Monette J, Noiseux $\mathrm{N}$, et al. Gait speed as an incremental predictor of mortality and major morbidity in elderly patients undergoing cardiac surgery. J Am Coll Cardiol 2010;56:1668-76.

13. Chen LK, Liu LK, Woo J, Assantachai P, Auyeung TW, Bahyah KS, et al. Sarcopenia in Asia: consensus report of the Asian Working Group for Sarcopenia. J Am Med Dir Assoc 2014;15:95-101.

14. Jang HC. How to diagnose sarcopenia in Korean older adults? Ann Geriatr Med Res 2018;22:73-9.

15. Graham JE, Ostir GV, Kuo YF, Fisher SR, Ottenbacher KJ. Relationship between test methodology and mean velocity in timed walk tests: a review. Arch Phys Med Rehabil 2008;89:865-72.

16. Jung HW, Kang MG, Choi JY, Yoon SJ, Kim SW, Kim KI, et al. Simple method of screening for frailty in older adults using a chronometer and tape measure in clinic. J Am Geriatr Soc 2018;66:157-60.

17. Won CW, Lee Y, Choi J, Kim KW, Park Y, Oh IH, et al. Starting construction of frailty cohort for elderly and intervention study. Ann Geriatr Med Res 2016;20:114-7.

18. Kim M, Won CW. Combinations of gait speed testing protocols (automatic vs manual timer, dynamic vs static start) can significantly influence the prevalence of slowness: results from the Korean frailty and aging cohort study. Arch Gerontol Geriatr 2019;81:215-21.

19. Lin LI. A concordance correlation coefficient to evaluate reproducibility. Biometrics 1989;45:255-68.

20. Ho KM. Using linear regression to assess dose-dependent bias on a Bland-Altman plot. J Emerg Crit Care Med 2018;2:68. 
76 Hee-Won Jung, et al.

21. Snijders AH, van de Warrenburg BP, Giladi N, Bloem BR. Neurological gait disorders in elderly people: clinical approach and classification. Lancet Neurol 2007;6:63-74.
22. Henriksen M, Lund H, Moe-Nilssen R, Bliddal H, DanneskiodSamsøe B. Test-retest reliability of trunk accelerometric gait analysis. Gait Posture 2004;19:288-97. 